\title{
Pharmacologically accelerating gastric emptying can reduce the calorie intake of obese individuals
}

$\mathrm{C}$ ontrary to popular belief, Silvia Delgado-Aros and colleagues from Barcelona have shown that the number of calories ingested by overweight or obese individuals can be decreased by increasing the rate at which the stomach empties nutrients into the small intestine.

While in the lab of Michael Camilleri at the Mayo Clinic, Delgado-Aros and co-workers saw that functional dyspepsia patients who emptied their stomachs more rapidly had worse symptoms (that is, failure to eat a normal-sized meal, feeling more full or sick after a meal). This suggested to Delgado-Aros that "rapid gastric emptying might be more relevant to the sensation of fullness and other postprandial symptoms than previously believed."

The knowledge that intestinal mucosal cells release neuropeptides when nutrients reach the small bowel, and that these neuropeptides contribute to the feeling of fullness or satiation, further encouraged the researchers in Barcelona. "We thought it was possible that the sooner these peptides are stimulated, the sooner an individual may feel full and this, presumably, could lead to stopping eating and reduce the amount of calories an individual can ingest within a meal," explains Delgado-Aros.
To test the hypothesis that pharmacological acceleration of the rate at which nutrients are emptied from the stomach into the small intestine induces precocious satiation and a reduction in food intake, the researchers recruited 30 overweight or obese volunteers who were otherwise healthy and had no gastrointestinal symptoms or disease.

Participants attended two study days, both of which followed the same protocol. On day 1 (baseline), all participants received an intravenous infusion of saline (placebo); on day 2 (1 week later) half of the participants were randomly allocated to receive an intravenous infusion of saline and half to receive an intravenous infusion of erythromycin $\left(3 \mathrm{mg} \mathrm{kg}^{-1}\right)$. To measure meal size and postprandial symptoms, all participants underwent a validated nutrient drink test on both study days. The radiolabeled liquid nutrient drink was consumed until participants were completely satiated and scintigraphy was used as a visual measurement of gastric nutrient emptying. Blood samples were also taken at baseline and during the assessment periods to measure various neuropeptide and hormone levels.

The amount of nutrients emptied in the first $15 \mathrm{~min}$ after initiation of eating

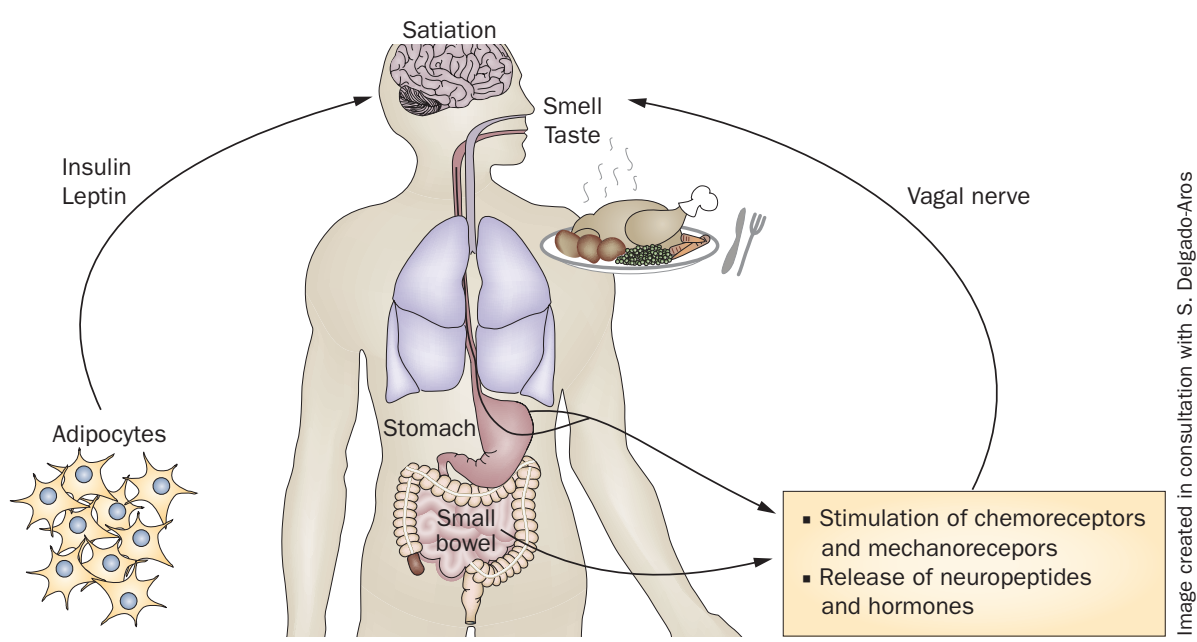

increased by $\sim 10 \%$ in those who received erythromycin. This acceleration translated into the consumption of 135 fewer Kcals when compared with those consumed by participants who received placebo. The sensation of fullness $30 \mathrm{~min}$ after eating was also more intense in the erythromycin group when compared with the placebo group, even though the group that received erythromycin had eaten less.

Plasma levels of satiation factors increased postprandially in both the erythromycin and placebo groups, but there were no significant differences between the two. The researchers suggest that the plasma levels might not adequately reflect changes in the satiation factors released from the small intestine. They also do not rule out a type II sampling error because of the exploratory nature of this part of their study.

"Prior to this study, most of the research performed in this field was directed to induce satiety by making the stomach stop emptying," says Delgado-Aros. "This study represents the proof of concept that accelerating the arrival of nutrients into the small bowel may be a useful mechanism to help reduce meal size in individuals who need help to control, or to modify, their eating habits."

As accelerated gastric emptying could be achieved only when erythromycin was administered intravenously, the researchers are now working to identify a drug that accelerates gastric emptying when administered orally. "We need to be sure that whatever drug we design for this is completely safe for the patient and has a 'pure' effect on gastric emptying," Delgado-Aros concludes. "There are no such drugs on the market right now."

Natalie J. Wood

Original article Torra, S. et al. Meal size can be decreased in obese subjects through pharmacological acceleration of gastric emptying. Int. J. Obes. doi:10.1038/ijo.2010.210 